\title{
Post-Dropping Behavior of Potato Aphids (Macrosiphum euphorbiae)
}

\author{
Rosalind K. Humphreys (D) Graeme D. Ruxton (D) \\ Alison J. Karley
}

Received: 19 April 2021 / Revised: 16 August 2021 / Accepted: 19 August 2021 / Published online: 25 August 2021

(C) The Author(s) 2021

\begin{abstract}
Dropping behavior is an effective antipredator defense utilized by many insects including aphids, which drop from plants to lower plant parts or underlying substrates to avoid attack from predatory invertebrates. While research commonly focusses on triggers of dropping, less attention is given to what happens to prey individuals following escape drops. In this study, the duration of tonic immobility, recovery rates, and cases of "instant recovery" (re-clinging to lower plant parts) exhibited by potato aphids (Macrosiphum euphorbiae) that dropped from potted seedlings in response to introduced ladybird (Adalia bipunctata) adults, lacewing (Chrysoperla carnea) larvae, and a standardized tactile stimulus were investigated in relation to a range of environmental factors. Air temperature had a negative correlation with the duration of post-dropping tonic immobility; as temperature increased, time spent motionless decreased. Aphids also showed a pattern of increased recovery rate at higher temperatures. Aphids may be selected
\end{abstract}

Supplementary Information The online version contains supplementary material available at https://doi. org/10.1007/s10905-021-09784-y.

R. K. Humphreys $(\bowtie) \cdot$ G. D. Ruxton

School of Biology, University of St Andrews, Dyer's Brae

House, St Andrews, Fife KY16 9TH, UK

e-mail: rosalindkh08@gmail.com

\section{A. J. Karley}

The James Hutton Institute, Invergowrie,

Dundee DD2 5DA, UK to move off the substrate quicker to avoid risks of overheating/desiccation at higher temperatures; and/ or higher body temperature facilitates locomotion. Stimulus type also influenced recovery rate back to the original seedling, with aphids generally recovering after the standardized stimulus quicker than after dropping triggered by a real predator. Considering cases of instant recovery onto lower-reaches of the host seedling, seedling height influenced the likelihood of re-clinging, with aphids that managed to instantly recover dropping from, on average, taller seedlings than aphids that dropped to the substrate. Plant architecture could mitigate the costs of dropping for aphids, but further studies quantifying understory foliage cover are needed.

Keywords Aphididae - Dropping behavior . Predator-prey interactions $\cdot$ Recovery $\cdot$ Tonic immobility

\section{Introduction}

Dropping is an important antipredator behavior, wherein a prey individual releases itself from a substrate (or ceases powered flight), passively or actively, such that gravity, wind or water currents power its subsequent escape from a perceived threat (reviewed in Humphreys and Ruxton 2019). Although a taxonomically widespread defense, dropping by insects has received by far the most attention (Humphreys 
and Ruxton 2019). But while considerable research efforts have developed understanding of on-plant triggers of dropping in insects, the behaviors individuals exhibit afterwards are comparatively neglected. When an insect drops from a plant onto the underlying substrate to avoid predation, it is exposed to many costs and risks associated with being off the plant, such as: lost feeding time, risk of desiccation, and potential exposure to new predators (Losey and Denno 1998a, b; Nelson 2007; Agabiti et al. 2016; Humphreys and Ruxton 2019). Yet, previous studies have observed insects behaving in ways that prolong the time spent off plants after dropping (see later). This exploratory study aims to investigate under-explored links between dropping and post-dropping behaviors.

Tonic immobility (hereafter referred to as TI) is one behavior that animals can exhibit post-dropping. TI describes the unlearned adoption of a motionless posture (sometimes a posture that resembles a dead individual, hence the alternative term "death-feigning") by prey individuals that is triggered by the presence of a perceived threat (Humphreys and Ruxton 2018). In insects, decreased ventilatory movements and the maintenance of a stiff posture are common during TI (Rogers and Simpson 2014); the adaptive function of this for individuals following an escape drop in response to a foliar-foraging predator may be reduced localization by other predators, such as birds or ground-foraging beetles that have detected the dropping movement (Wohlers 1981; Miyatake et al. 2009; Humphreys and Ruxton 2018). A few studies have found that the duration of TI in dropped insects can vary depending on a range of factors, including the nature of the stimulus that triggered dropping (Wohlers 1981), height dropped from (Niku 1975), and ambient temperature (Miyatake et al. 2008); but much remains unexplored. Following TI, normal behavior can rapidly resume (Rogers and Simpson 2014), but the mode of recovery of dropped insects back to host plants shows variation. For example, some aphids (family Aphididae) undertake different characteristic movements after post-drop TI: individuals either run slowly but show a high turning rate (deemed "searchers") or run quickly but show a low turning rate (deemed "runners") (Niku 1975, 1976; Roitberg et al. 1979; Ben-Ari et al. 2015). A limited number of studies exploring post-drop recovery suggest that the nature of the stimulus that prompted dropping may influence the type of movement shown during recovery (Phelan et al. 1976; Wohlers 1981), but this requires further study and the influence of other factors on the rate at which dropped insects return to plants is poorly understood. For any insect that has dropped to the substrate from a plant, the nature of any TI and recovery behaviors it exhibits will have important consequences for the costs it experiences, its survival likelihood, and overall fitness.

Of course, the most effective way of mitigating the costs of dropping is to avoid falling all the way to the substrate in the first instance. Many flightless insects exhibit an "aerial righting" reflex, wherein they change their body posture in such a way - after releasing from a substrate - that their ventral side and any adhesive organs are directed downwards (Yanoviak et al. 2009; Jusufi et al. 2011; Ribak et al. 2013; Meresman et al. 2014; Zeng et al. 2017). This enables re-grasping of plant material underlying the position they dropped from, so that they do not fall to the ground after dropping (Nelson 2007; Ribak et al. 2013); we also refer to this as "instant recovery" back to the host plant. Individuals' reaction to detachment from plants and aerial righting take time, and so it is unsurprising that the vertical position on the plant from which an insect drops appears to influence the likelihood of re-clinging (Ribak et al. 2013; Meresman et al. 2017). Additionally, several characteristics of host plants have been found to relate to the success of dropped insects in re-clinging, including leaf architecture (Matsubara and Sugiura 2018), foliage density, and full plant height (Meresman et al. 2017), but the link between dropping and this important costmitigating behavior remains under-explored.

This present study aims to highlight often-underappreciated post-dropping behavior as an important aspect of predator-prey interactions. Potato aphids (M. euphorbiae), our study species, are one of many commercially-important aphid species that use dropping as a significant component of their antipredator behavioral repertoire (Humphreys et al. 2021). They can colonize potato plants at an early developmental stage, and the colony can go through a number of generations during the life cycle of the plant (AHDB 2015). Here, we explored potato aphids' post-dropping behavior (TI, recovery, and re-clinging ability or "instant recovery") using additional data collected during a study exploring responses to foraging predators on live, immature potato plants (which we refer 
to as "seedlings" throughout, for ease of writing). In examining TI, our interest was its duration rather the specific form exhibited. When considering recovery, we did not focus on the mechanism of recovery (i.e., the movements or walking routes of aphids), rather on time taken. These design decisions were taken because we are interested in investigating dropping from a behavioral-ecology framework (instead of its mechanics), by considering the benefits - i.e. survival - against the time spent off-plant, which can be presumed to correlate with associated costs; such as lost feeding time, risk of desiccation, and time exposed to other potential risks on the substrate (Losey and Denno 1998a, b; Nelson 2007; Agabiti et al. 2016; Humphreys and Ruxton 2019). Potato aphids' TI and recovery rates were opportunistically explored in relation to a range of different factors: stimulus type; substrate; maximum height of seedlings; distance dropped from original host seedling; and ambient temperature. Some of these variables have been previously explored in other insect species, but not potato aphids; and no study in any species has systematically explored them in concert. The ability of potato aphids to re-grasp underlying plant material after dropping was explored in relation to several plant-related factors: the position on seedlings that aphids dropped from (that is, the type of plant section an aphid was located on, e.g. stem, petiole, leaf upperside, leaf underside); seedling age; and the maximum height of seedlings (similar to the "field height" measure used by Meresman et al. 2017). Because this exploratory study considers many factors for each post-dropping behaviour, we detail our specific predictions and the rationale behind them in Table 1 .

\section{Materials and Methods}

Post-dropping behavior of 8-9 d old 3rd-4th instar potato aphids was recorded following encounters with lacewing larvae (Chrysoperla carnea) and adult ladybirds (Adalia bipunctata) on live, potted seedlings 2-3 weeks from planting (as a reminder, although these immature potato plants were technically grown from tubers, we use the term "seedlings" throughout for ease of writing). The experiment took place in a glasshouse at the James Hutton Institute (Dundee, UK), during July 2019. Half of the pots in the experiment were left with sand-perlite-peat compost mix (referred to as "soil" throughout) as the substrate and half had a thin top layer of fine white Scalare river sand (grains $<0.5 \mathrm{~mm}$ ) added, determined randomly for any given assay. The maximum height of the seedlings in any given pot was recorded to the nearest $0.5 \mathrm{~cm}$, as well as the seedlings' age since potting (in days). Pots were then infested in the late afternoon and left overnight with 16 wingless immature potato aphids. Assays were then conducted, wherein a predator was introduced to forage on the seedlings in a pot for $30 \mathrm{~min}$; this predator was replaced as needed but there was only ever one predator present on a pot (see Supplementary Material S1 for details of insect rearing and the experimental protocol). The temperature (to within $0.5^{\circ} \mathrm{C}$ ) was recorded using a Brannan "Push button Minimum/Maximum" thermometer, immediately before the first predator was introduced during filming. The predator's foraging period was filmed from overhead with a camcorder fixed to a tripod. As the predator moved about seedlings, details of any dropping behavior exhibited by aphids apparently in response to the predator's presence were dictated to the camera by the experimenter; this included drops from the predator's current seedling section and on nearby sections. When an aphid dropped, the following additional metrics were recorded, if observed: which predator type triggered the drop, what seedling position the aphid dropped from (considered as stem, petiole or petiolule or lateral stem, leaf upperside, or leaf underside), the time the aphid dropped, the horizontal distance the aphid landed from the stem of its original host seedling (to the nearest $0.1 \mathrm{~cm}$, measured either with a flexible paper ruler during trials or calculated from the planar view of recordings post-trials), the time the aphid moved again after any period of TI, and the time when the aphid subsequently had all its legs back onto a seedling following recovery. Any cases of aphids re-clinging to underlying plant parts after dropping, rather than falling to the substrate, were also observed and their occurrence dictated to the camera by the experimenter. The filmed period described above constituted Part 1 of data collection.

To further explore the post-dropping tendencies of potato aphids, two additional parts followed Part 1 of assays. Following the removal of the predator, filming continued and temperature was recorded for a second 


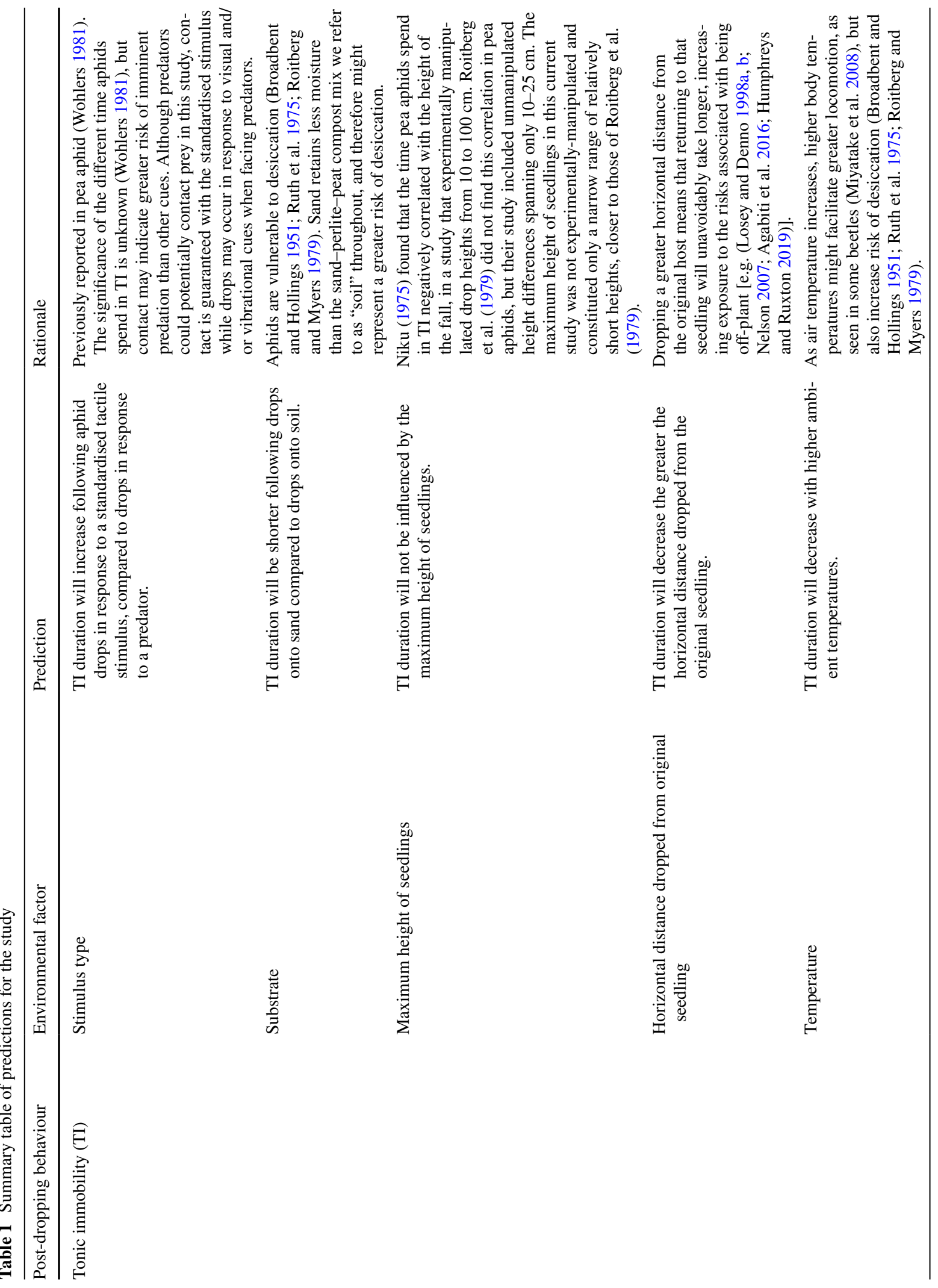




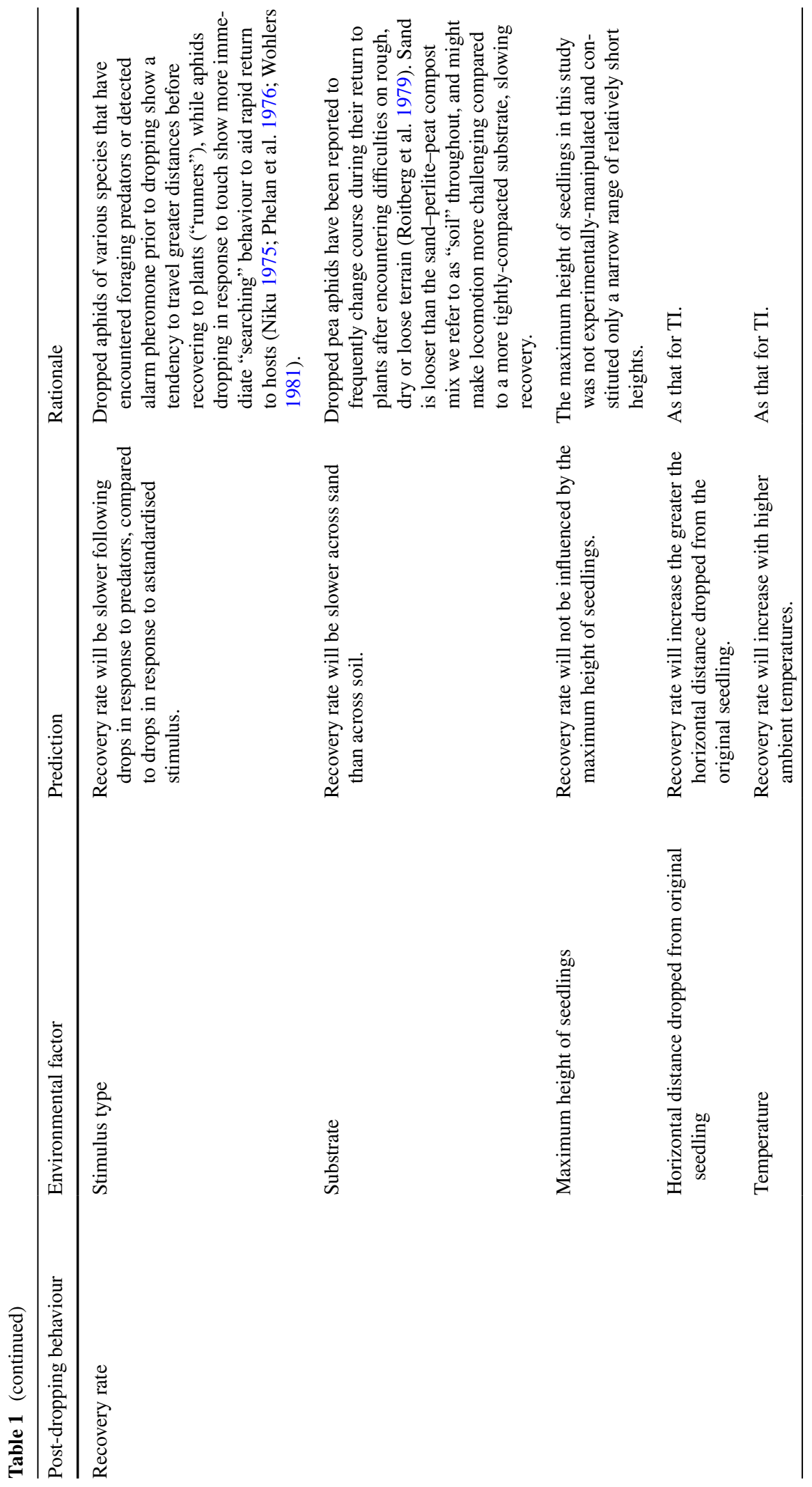




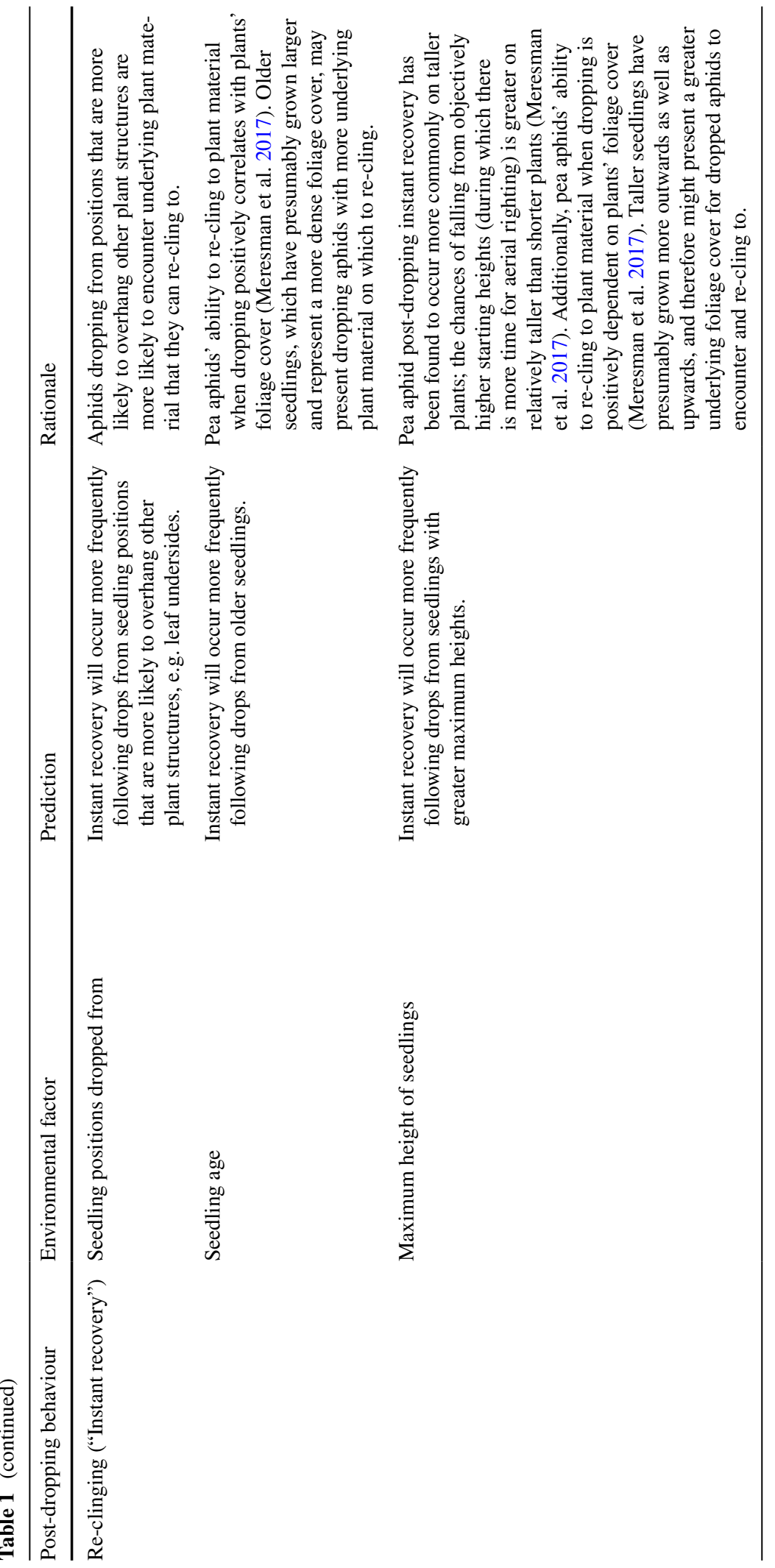


time. For Part 2 of trials, then, each individual aphid remaining on the seedlings - that is those that had either not left their original host seedling during the predator foraging period, or those that had left (either via walking or dropping) but had then re-settled on one of the seedlings prior to the end of Part 1 - was subjected to an initial bout of standardized mechanical stimulus. The standardized stimulus consisted of a brief and gentle stroke from a small, fine paintbrush (similar to Wohlers 1981) running from head-to-tail and just making contact with the individual. If any aphids dropped in response to this contact, the relevant metrics listed above were recorded, so long as the associated behaviors occurred within $5 \mathrm{~min}$ of the standardized stimulus (after this time the recording was stopped). After Part 2, pots were left for $30 \mathrm{~min}$ in order to allow the aphids to recover. After this time, the video camera was set to record again and the temperature was recorded for a final time. Part 3 then consisted of a second bout of standardized stimulus and recording of dropping responses and post-dropping behaviors, carried out on any aphids that were still settled or had re-settled on seedlings in the same way as in Part 2.

Thirty-two assays, 16 with each predator type in Part 1, took place with 8-9 d old 3rd-4th instar potato aphid nymphs using pots each with two 2-3-weekold potato cv. Desirée seedlings (see Supplementary Material S1 for more details of methods). Behavioral Observation Research Interactive Software (BORIS) (Friard and Gamba 2016) was used to watch the video footage, code behaviors, record their timings, and measure some distances aphids dropped from their original host seedling. From the time and distance measures, post-dropping TI durations and (only where aphids returned to their original host seedlings) recovery rates $(\mathrm{cm} / \mathrm{s})$ were calculated. All statistical analyses were conducted using $\mathrm{R}$ version 4.0.3 ( $\mathrm{R}$ Core Team 2020).

\section{Results}

Post-Drop Tonic Immobility (TI) Duration

Data from Part 1 of the experiment, during the predator foraging period, were used to explore whether different variables affect the duration of TI potato aphids undergo post-dropping. Only drops where either a ladybird adult or lacewing larva was considered to be the stimulus were included, as the focus of the study is behavior following antipredator dropping. The only exception to this was when stimulus type more broadly - rather than predator type alone - was considered and, in this case, data from Parts 2 and 3 of the experiment were also utilized. For all analyses concerning TI, cases of instant recovery and cases where aphids fell off plant pots entirely were excluded (though see Supplementary Material S2 for details of $\mathrm{TI}$ in cases of instant recovery).

\section{Stimulus Type}

During Part 1 of trials, the duration of TI was recorded for 48 of the 51 cases where ladybird adults appeared to trigger potato aphids to drop onto the underlying substrate. TI durations were recorded for all 22 of the cases where lacewing larvae caused aphid drops to the substrate. Comparing the mean durations of TI, no significant difference was found between aphids that dropped in response to ladybirds (mean \pm standard deviation [hereafter sd]: $11.5 \pm 25.1 \mathrm{~s}$ ) and those that dropped in response to lacewings (mean $\pm \mathrm{sd}$ : $4.7 \pm 5.1 \mathrm{~s}$ ) (Welch twosample t-test: $\mathrm{t}=1.8, \mathrm{df}=54.9, \quad P=0.078)$. The same was true in a comparison of median TI duration (median \pm interquartile range [hereafter IQR] for ladybird-induced drops: $2.1 \pm 4.6 \mathrm{~s}$, median $\pm \mathrm{IQR}$ for lacewing-induced drops: $2.8 \pm 5.0 \mathrm{~s}$, Wilcox test $\mathrm{W}=549, P=0.80$ ).

Parts 2 and 3 of trials were then considered in order to test whether the duration of TI differed significantly depending on whether aphids were faced with natural predators or standardized stimuli. Both predator types were combined for this analysis, due to the lack of significant difference in TI duration reported above, and compared with the TI durations recorded following drops caused by the first standardized stimulus (immediately after the predator foraging period in Part 1) and the second standardized stimulus (following a 30-min recovery period after Part 2) separately. Post-drop TI durations were recorded for: 70 of the 73 times aphids dropped in response to predators, 108 of the 111 times aphids dropped in response to the first standardized stimulus, and 89 of the 92 times aphids dropped in response to the second standardized stimulus. A one-way ANOVA found no significant difference in the mean duration 
of TI of aphids dropping as a result of the different stimuli (mean \pm sd for predator: $9.3 \pm 21.1 \mathrm{~s}$, first standardized stimulus: $6.7 \pm 12.4 \mathrm{~s}$, second standardized stimulus: $14.5 \pm 43.2 \mathrm{~s}, \mathrm{~F}=1.69$, num $\mathrm{df}=2.00$, denom df $=129, P=0.19)$. Similarly, a Kruskal-Wallis rank sum test found no significant difference in the median durations either (medians $\pm \mathrm{IQR}$ for predator: $2.1 \pm 4.8 \mathrm{~s}$, first standardized stimulus: $2.3 \pm 6.7 \mathrm{~s}$, second standardized stimulus: $2.6 \pm 7.8 \mathrm{~s}$, KruskalWallis chi-squared $=0.22909, \mathrm{df}=2, \quad P=0.8918$ ). Any such comparisons must be interpreted cautiously, since individuals were of course not randomized to stimulus type, and thus confounding factors may be important.

\section{Substrate}

TI duration was recorded for 35 of the 37 of the predator-induced drops that resulted in aphids landing on sand as the underlying substrate, and 35 of the 36 cases where aphids landed on soil. A Welch twosample $\mathrm{t}$-test found no significant difference $(\mathrm{t}=1.28$, $\mathrm{df}=41.0, P=0.21)$ in the mean duration of TI of dropped aphids on sand (mean $\pm \mathrm{sd}: 12.5 \pm 28.3 \mathrm{~s}$ ) compared to those that dropped on soil (mean $\pm \mathrm{sd}$ : $6.1 \pm 9.1 \mathrm{~s})$. Similarly, a Wilcox test found that there was also no significant difference $(\mathrm{W}=468, P=0.09)$ in median duration of TI of dropped aphids on sand (median \pm IQR: $1.4 \pm 4.5 \mathrm{~s}$ ) compared to those that dropped on soil (median \pm IQR: $3.3 \pm 5.5 \mathrm{~s}$ ).

\section{Maximum Height of Seedlings}

Previous studies have reported contrasting findings that height dropped from does (Niku 1975) and does not influence aphid TI duration (Roitberg et al. 1979), and so height was explored further here in another context where plant height was not experimentally manipulated. The relationship between the height of seedlings and the duration of aphid TI post-dropping was explored for the 70 cases where TI duration was recorded (73 drops were observed across Part 1). The maximum height of seedlings ranged from 9.0-30.0 cm (mean: $15.1 \mathrm{~cm}$, median: $11.0 \mathrm{~cm}$ ), and TI durations ranged from $0.4-133.9 \mathrm{~s}$ (mean: $9.3 \mathrm{~s}$, median: $2.1 \mathrm{~s}$ ). A Pearson's product-moment correlation test found no significant correlation between maximum height of seedlings and aphids' post-drop TI durations $(\mathrm{t}=0.08, \mathrm{df}=68, P=0.94)$.
Horizontal Distance Dropped from Original Seedling

The relationship between the horizontal distance dropped aphids landed from their original host seedling and the duration of their subsequent TI was explored for the 67 cases where both the distance and TI duration were recorded. The distance landed from host seedling stems ranged from $0.2-9.0 \mathrm{~cm}$ (mean: $3.0 \mathrm{~cm}$, median: $3.0 \mathrm{~cm}$ ), and TI durations ranged from 0.4-133.9 s (mean: $9.7 \mathrm{~s}$, median: $2.4 \mathrm{~s}$ ). A Pearson's product-moment correlation test found no significant correlation between distance landed from original host seedling and subsequent TI durations $(\mathrm{t}=-0.21, \mathrm{df}=65, P=0.84)$.

\section{Temperature}

The relationship between the glasshouse temperature at the start of Part 1 and the duration of aphid TI post-dropping was explored for the 70 cases where TI duration was recorded. Temperatures ranged from 22 - $40^{\circ} \mathrm{C}$ (mean: $29.9^{\circ} \mathrm{C}$, median: $29.5^{\circ} \mathrm{C}$ ); descriptive statistics for TI durations are given in the "Maximum Height of Seedlings" section above. A Pearson's product-moment correlation test found a significant negative correlation between the temperature recorded at the beginning of the predator foraging period and the duration of aphids' TI post-dropping $(\mathrm{t}=-2.2$, $\mathrm{df}=68, P<0.05)$. At higher glasshouse temperatures, aphids appeared to spend less time motionless on the substrate (Fig. 1).

\section{Post-Drop Recovery Rates Back to Original Host} Seedlings

The recovery of aphids considered in these analyses refers to activity occurring subsequent to any period of TI recorded post-dropping. The rate of recovery $(\mathrm{cm} / \mathrm{s})$ was calculated for all aphids that returned to their original seedling after landing on the substrate, using the time they started moving again after any TI, the time they had all their feet back on their original seedling, and the approximate horizontal distance they landed at from the stem of their original seedling. Importantly, the path the aphid followed - whether it was straight or torturous - was not recorded. Therefore, while distance was measured as a straight line and rate of recovery was calculated as distance over time, this does not guarantee that the 


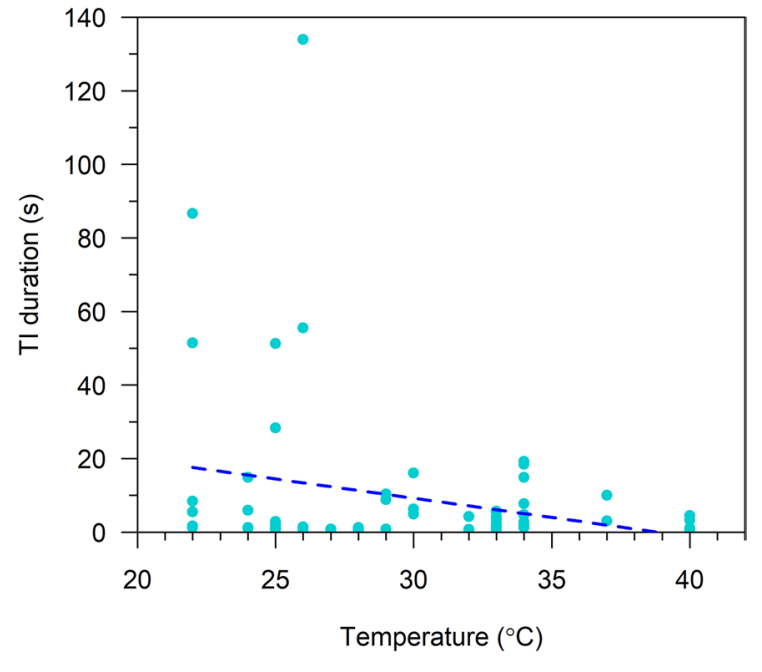

Fig. 1 As the glasshouse temperature recorded at the beginning of Part 1 increased, the duration of dropped potato aphids' tonic immobility (TI) on the underlying substrate appeared to decrease $(n=70)$

aphid moved directly back to its original host seedling. An aphid may have moved quickly across the substrate, but looped around on the substrate before its return, so although its speed across the substrate might have been great its rate of recovery may have been relatively slow. We focus on recovery rate as an informative measure regarding the relative time and energy costs we can assume aphids suffer from being off plants not feeding. As with the TI analyses, data from exclusively Part 1 of the experiment was used to explore whether different variables affect aphid recovery rate post-dropping, with the exception of the broader stimulus analyses; for which, data from Parts 2 and 3 of the experiment were also utilized. Also, cases of instant recovery and cases where aphids fell off plant pots entirely were again excluded.

\section{Stimulus Type}

For Part 1, the rate of recovery was calculated for all 23 instances where aphids dropped in response to a foraging ladybird adult, and for eight of the nine instances where aphids dropped in response to a foraging lacewing larva. No significant difference was found (Welch two-sample t-test: $\mathrm{t}=-1.06, \mathrm{df}=8.6$, $P=0.32$ ) between the mean (mean \pm sd for ladybirdinduced: $\quad 0.071 \pm 0.060 \mathrm{~cm} / \mathrm{s}$, lacewing-induced: $0.114 \pm 0.107 \mathrm{~cm} / \mathrm{s}$ ) or median (median $\pm \mathrm{IQR}$ for ladybird-induced: $\quad 0.063 \pm 0.065 \mathrm{~cm} / \mathrm{s}$, lacewinginduced: $0.101 \pm 0.167 \mathrm{~cm} / \mathrm{s}$, Wilcox test $\mathrm{W}=76$, $P=0.49$ ) recovery rate of aphids dropping in response to the different predatory stimuli.

Parts 2 and 3 of trials were then considered to explore whether aphids' recovery rate back to their original host seedlings significantly differed depending on whether dropping had been provoked by a foraging predator (as with the TI analyses, data for ladybird adults and lacewing larvae were combined), a first standardized stimulus (Part 2), or a second standardized stimulus (Part 3). Subsequent recovery rates $(\mathrm{cm} / \mathrm{s})$ were calculated for 31 of the 32 drops in response to predators that were followed by a return to the original host seedling, 24 of the 25 drops in response to the first standardized stimulus that were followed by a return to the original host seedling, and all 25 drops in response to the second standardized stimulus that were followed by a return to the original host seedling. A Kruskal-Wallis rank sum test found a significant difference in the median recovery rate of aphids dropping in response to different stimuli (median \pm IQR for predator: $0.068 \pm 0.078 \mathrm{~cm} / \mathrm{s}$, first standardized stimulus: $0.121 \pm 0.085 \mathrm{~cm} / \mathrm{s}$, second standardized stimulus: $0.123 \pm 0.108 \mathrm{~cm} / \mathrm{s}$, KruskalWallis chi-squared $=9.2981$, df $=2, P<0.01$ ). Additionally, a one-way ANOVA found a significant difference in the mean recovery rate of aphids dropping in response to different stimuli (mean \pm sd for predator: $0.082 \pm 0.075 \mathrm{~cm} / \mathrm{s}$, first standardized stimulus: $0.141 \pm 0.081 \mathrm{~cm} / \mathrm{s}$, second standardized stimulus: $0.141 \pm 0.124 \mathrm{~cm} / \mathrm{s}, \quad F=4.5672$, num $\mathrm{df}=2.000$, denom $\mathrm{df}=45.641, P<0.05)$. A post-hoc GamesHowell test (suitable for unequal variances) then found a significant difference between recovery rate following drops triggered by predators and drops triggered by the first standardized stimuli $(P<0.05)$. Potato aphids appeared to recover back to their original host seedling at a slower rate when their drop from that seedling had been provoked by the presence of a natural predator, compared to when their drop had been triggered by contact from the standardized stimulus (Fig. 2).

\section{Substrate}

Recovery rate was recorded for all 13 cases in Part 1 where aphids recovered back to their original seedlings after dropping to sand as the underlying 


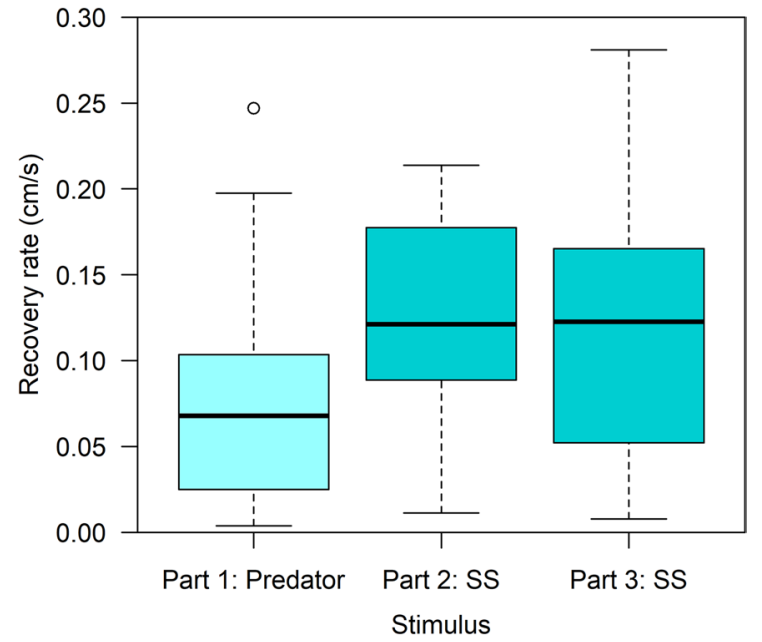

Fig. 2 The recovery rates $(\mathrm{cm} / \mathrm{s})$ of potato aphids (excluding any periods of tonic immobility) that recovered back to their original host seedling, following: drops provoked by natural predators (ladybird adults and lacewing larvae) in Part 1 $(n=31)$, drops provoked by the first bought of standardized stimulus (SS) in Part $2(n=24)$, and drops provoked by the second bought of SS in Part $3(n=25)$. The SS consisted of a brief and gentle head-to-tail stroke from a fine paintbrush

substrate, but for only 18 of the 19 cases where the underlying substrate was soil. No significant difference (Welch two-sample t-test: $t=-1.5663$, $\mathrm{df}=28.025, P=0.1285$ ) was found in the mean (mean \pm sd for sand: $0.060 \pm 0.051 \mathrm{~cm} / \mathrm{s}$, soil: $0.099 \pm 0.087 \mathrm{~cm} / \mathrm{s}$ ) or median (median $\pm \mathrm{IQR}$ for sand: $0.041 \pm 0.061 \mathrm{~cm} / \mathrm{s}$, soil: $0.072 \pm 0.115 \mathrm{~cm} / \mathrm{s}$, Wilcox test $\mathrm{W}=92, P=0.3318$ ) recovery rate of aphids dropping onto the different substrates.

\section{Maximum Height of Seedlings}

The relationship between the height of seedlings and the recovery rate of aphids was explored for the 31 cases where aphids returned to their original host seedlings following antipredator dropping and all necessary time and distance measures were recorded (32 drops where aphids returned to their original host seedlings were observed in total across Part 1). The maximum height of seedlings in these trials ranged from 9.0-25.0 cm (mean: $13.7 \mathrm{~cm}$, median: $11.0 \mathrm{~cm}$ ), and recovery rates ranged from $0.004-0.305 \mathrm{~cm} / \mathrm{s}$ (mean: $0.082 \mathrm{~cm} / \mathrm{s}$, median: $0.068 \mathrm{~cm} / \mathrm{s}$ ). A Pearson's product-moment correlation test found no significant correlation between maximum height of seedlings and aphids' post-drop recovery rates back to their original host seedlings $(\mathrm{t}=-1.0235, \mathrm{df}=29$, $P=0.3146)$.

\section{Horizontal Distance Dropped from Original Seedling}

The relationship between the horizontal distance dropped aphids landed from their original host seedling and the rate of their recovery back to that seedling was explored for the 31 cases where all necessary time and distance measures were recorded. The distance landed from host seedling stems ranged from 0.2-6.0 cm (mean: $2.4 \mathrm{~cm}$, median: $2.5 \mathrm{~cm}$ ); descriptive statistics for recovery rates are given in the "Maximum Height of Seedlings" section immediately above. A Pearson's product-moment correlation test found no significant correlation between straight horizontal distance landed from original host seedling and subsequent rate of recovery $(\mathrm{t}=0.15257, \mathrm{df}=29$, $P=0.8798)$.

\section{Temperature}

The relationship between the glasshouse temperature at the start of Part 1 and the recovery rate of aphids was explored for the 31 cases where aphids returned to their original host seedlings following antipredator dropping and recovery rate could be calculated. Temperatures ranged from $22-40^{\circ} \mathrm{C}$ (mean: $30.5^{\circ} \mathrm{C}$, median: $33.0^{\circ} \mathrm{C}$ ); descriptive statistics for recovery rates are given above. A Pearson's product-moment correlation test found a significant positive correlation between the temperature recorded at the beginning of the predator foraging period and the rate of aphids' recovery back to their original host seedlings $(\mathrm{t}=2.6046, \mathrm{df}=29, P<0.05)$. At higher glasshouse temperatures, aphids appeared to return to seedlings at a relatively faster rate (Fig. 3).

\section{Mitigation of Dropping/Re-Clinging/Instant Recovery}

Here we consider cases where aphids landed on an underlying section of their current seedling following a drop (what we deem instant recovery) across all 3 parts of the experiment - this may be one means by which aphids can mitigate the costs associated with dropping. 31 cases of instant recovery occurred in total, with 7 following drops triggered by predator 


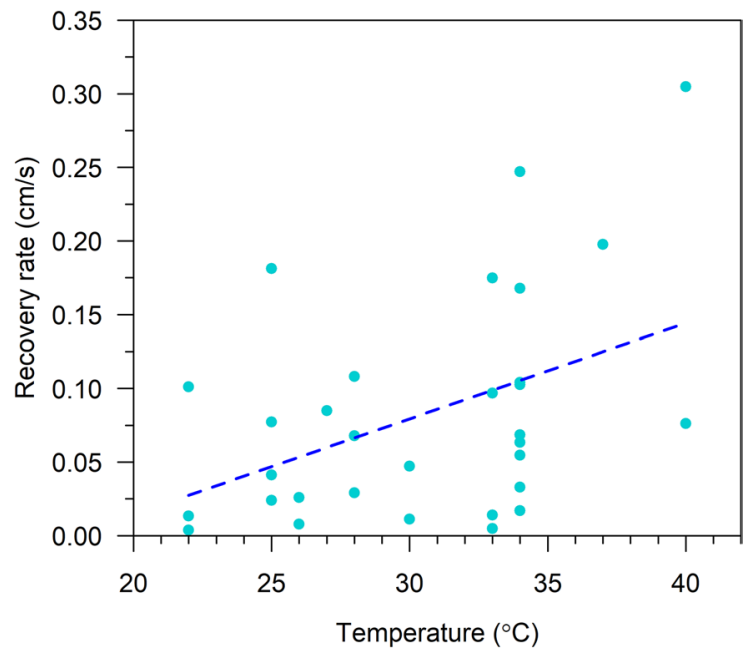

Fig. 3 As the glasshouse temperature recorded at the beginning of Part 1 increased, the post-drop rate of recovery of potato aphids that recovered back to their original host seedlings increased $(n=31)$

contact ( 6 by ladybird adults and 1 by a lacewing larva) and 24 following drops triggered by the standardized stimulus (11 by the first standardized stimulus and 13 by the second).

\section{Seedling Positions Dropped from}

Thirty of the 31 observed cases of instant recovery followed drops from leaf undersides, with the other one occurring following a drop from a seedling's main stem.

\section{Seedling Age and Maximum Height of Seedlings}

Using data from all 31 cases of instant recovery, a chi-squared test for given probabilities found no significant difference between the proportion of instant recovery following drops and the proportion of total drops that occurred on experimental pots with seedlings of different ages $\left(\chi_{8}^{2}=0.28, P=1\right)$. Seedling ages in this experiment, however, only ranged from 18 to $26 \mathrm{~d}$ old and did not consistently reflect relative heights or foliage cover. To explore another plant factor, then, the maximum heights of seedlings in pots on which instant recovery occurred (range: 10.0-30.0 cm, mean: $17.4 \mathrm{~cm}$, median: $18.0 \mathrm{~cm}$ ) were then compared with the maximum heights of seedlings in pots across cases of dropping where

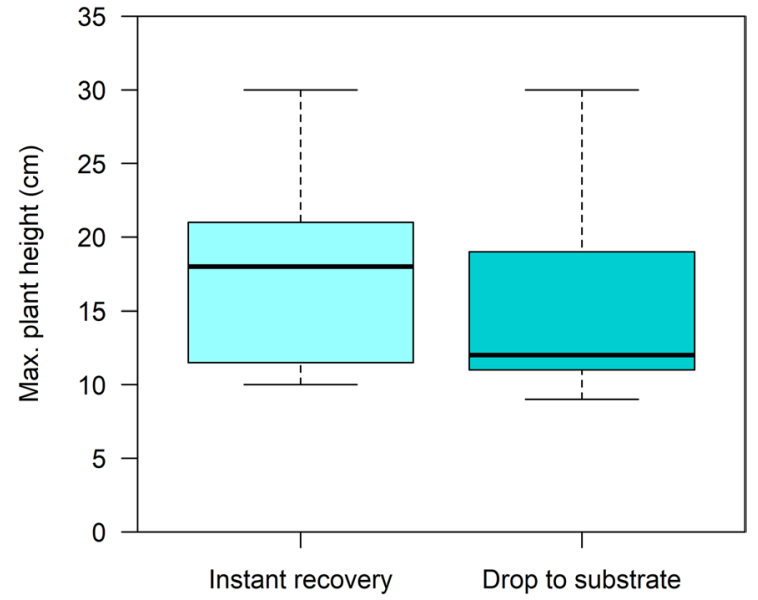

Fig. 4 The maximum height of seedlings in pots on which cases of instant recovery (where aphids landed on an underlying section of their current plant following a drop) occurred $(n=31)$, and the maximum height of seedlings in pots on which aphids dropped to the substrate $(n=313)$ across all three parts of the experiment. The maximum seedling height for any given trial occurred in the data the same number of times that instant recovery or dropping to the substrate occurred during that trial. Instant recovery appeared to occur more frequently following drops from seedlings with a greater maximum height compared to the maximum heights of seedlings from which dropping to the substrate occurred

instant recovery did not occur and aphids fell to the underlying substrate (range: 9.0-30.0 cm, mean: $15.02 \mathrm{~cm}$, median: $12.0 \mathrm{~cm}$ ). Because cases of dropping occurred in all trials, all of the seedlings on which instant recovery occurred appeared in both groups (this is seen in the considerable overlap of value ranges in Fig. 4). But consideration of the maximum heights of seedlings in pots on which no instant recovery occurred and the relative frequency of cases of instant recovery or drops to the substrate on any individual pair of seedlings could still be informative, despite the measure being crude. For this reason, the maximum seedling height for each given trial was repeated in the data for the number of times that instant recovery or dropping to the substrate occurred, so there were a total of 31 heights listed for the instant recovery data and 313 heights for the drops to the substrate data. No significant difference was found between the medians, though the $\mathrm{p}$-value was verging on significance (Wilcox test $\mathrm{W}=5876, P=0.05$ ), but a Welch two-sample t-test found a significant difference between the means $(\mathrm{t}=2.15, \mathrm{df}=35.8, P<0.05)$. The mean maximum height of seedlings in pots on 
which instant recovery was seen to occur following drops was significantly greater than that of seedlings in pots on which instant recovery did not occur following dropping.

\section{Discussion}

Overall, potato aphids were found to vary in their post-dropping behavior, indicating that these behaviors occur flexibly under different conditions; they are an important aspect of predator-prey interactions where dropping is an option within aphids' defensive repertoires. Over the course of the 32 assays, there were 17 instances of aphids being grabbed and consumed by foraging predators while on seedlings; no aphids that dropped to the substrate were ever pursued by the predator that triggered the drop. Although we explored the influence of a range of variables on post-dropping TI and recovery rates, it is unlikely that the significant results we detected were due to type I error. The effects we detect were always in the predicted directions and the associated p-values were never marginal. To the best of our knowledge, our study provides novel findings regarding the significant influence of several factors on potato aphids' TI and recovery rates post-dropping, as well as the first data collected on influencers of their re-clinging abilities.

\section{Post-Drop Tonic Immobility (TI) Duration}

A striking aspect of post-dropping behavior was that droppers often showed a period of stillness (akin to tonic immobility) prior to moving across the substrate to recover to a seedling. As predicted (see Table 1), temperature had a significant negative correlation with the duration for which potato aphids exhibited TI after dropping. As the underlying substrate gets hotter, the risk of overheating and desiccation increases for aphids (Broadbent and Hollings 1951; Ruth et al. 1975; Roitberg and Myers 1979; Dill et al. 1990), and therefore dropped aphids may be selected to reduce the time spent in an immobile state and increase activity that will result in escape from the substrate. Further, seed beetles have been shown to exhibit TI less strongly at higher ambient temperatures, a finding which Miyatake et al. (2008) attributed to running away being a more viable energetic option under such conditions compared to when ambient temperatures were low. It is possible that lower temperatures may also, to some extent, reduce the viability of aphids running away or prevent them from reaching their highest possible running speeds (and thereby making TI a more practicable defensive alternative). However, the post-dropping response of aphids to temperature is complicated by metabolic effects, the risk of damage at high levels, and the issue of left-censored data. Further studies purpose-designed to manipulate temperatures and test greater sample sizes of TI durations across a controlled range of temperatures are needed to explore the effect of temperature on potato aphid behaviors further.

The duration of TI exhibited by potato aphids did not appear to be significantly affected by the other variables explored in this experiment. While Wohlers (1981) found that pea aphids exhibited TI for longer when dislodged by tactile stimuli compared to those which dropped in response to an approaching coccinellid predator, potato aphids in this current study did not significantly differ in TI durations depending on whether they dropped in response to a foraging predator or the standardized stimulus (going against our prediction in Table 1). This difference may be due to the fact that in this study the vast majority of drops were triggered by contact with the predator, whereas for Wohlers (1981) the visual and/or vibrational cues of an approaching coccinellid predator were sufficient stimuli to trigger dropping. Perhaps the tactile standardized stimulus in this study was interpreted by aphids as being a similar threat as a predator, as both involved direct contact, and therefore provoked similar TI durations. The contrast in findings might also represent a species-specific difference between pea and potato aphids, perhaps resulting from their different life histories and interactions with natural enemies (van Emden and Harrington 2007; AHDB 2015). It remains uncertain why different TI durations on the substrate would theoretically be adaptive based on the nature of the stimulus experienced on the plant, given that foliar-foraging predators do not tend to follow dropped aphids to the ground (Wohlers 1981). Contrary to expectations (see Table 1), the TI durations of potato aphids also did not significantly differ depending on the substrate on which they landed. Heat stress poses a threat of desiccation to aphids (Broadbent 
and Hollings 1951; Ruth et al. 1975; Roitberg and Myers 1979), and different substrates will heat up and retain moisture to varying extents; we had assumed here that sand may represent a drier, more dangerous substrate. However, as these properties were not measured in the sand and soil substrates used in this current experiment, we cannot be certain that these substrates represented significantly different threats to desiccation. Future studies quantifying the moisture content and temperatures of differing substrates and examining the desiccation risk they pose to dropped aphids are needed in order to test the possible influence of substrate on TI duration further. The maximum height of seedlings dropped from also did not significantly influence the duration of time TI was exhibited, as we predicted (see Table 1). Niku (1975) found that pea aphids exhibit TI for different durations depending on height fallen, in a study that varied drops between 10 and $100 \mathrm{~cm}$. TI duration was negatively correlated with the height fallen (Niku 1975), possibly resulting from the fact that it is less likely that predators could follow aphids' trajectory or otherwise pursue them if they fall a greater distance, and therefore there is less of a need to remain immobile to reduce predator localization. However, Roitberg et al. (1979) did not find this correlation in their study where pea aphid drops varied in height - by chance - between 10 and $25 \mathrm{~cm}$; the difference in these heights may not have been big enough to translate into significant differences in predators' tracking abilities of dropped prey. In this current study, potato aphid drops took place from seedlings whose maximum heights ranged from 9 to $30 \mathrm{~cm}$ (closer to the range of Roitberg et al. 1979) and the specific distances fallen by individuals from particular seedling parts could not be recorded accurately without disturbing the set-up during assays; for these reasons, it is not surprising that we also found no significant relationship. Finally, we found no significant correlation between the horizontal distance dropped from the original host seedling and the time dropped aphids spent in a state of TI. Although we had predicted that greater distances may mean aphids are selected to reduce desiccation risk by rapidly resuming motion towards seedlings again (see Table 1), more relevant variables that would impact how vulnerable aphids assess themselves as being on the substrate include horizontal distance from any plant (rather than their original host alone), light conditions and shading, and the detectability of plants that could be recovered to.

\section{Post-Drop Recovery Rates Back to Original Host Seedlings}

In line with our prediction (see Table 1), aphids showed a pattern of increased recovery rate back to the original host seedlings with higher temperatures. Combined with the finding above that aphids' TI durations decreased at higher temperatures, this strongly suggests that post-dropping behavior is adapted to reduce the time spent on the substrate when it is hotter; this makes sense given the risk of desiccation associated with time spent exposed on hot and dry substrate (Broadbent and Hollings 1951; Ruth et al. 1975; Roitberg and Myers 1979; Dill et al. 1990). Alternatively, or additionally, in both cases higher temperatures also likely increase insect body temperatures, facilitating greater activity and more rapid locomotion. The recovery rate of potato aphids was also affected by stimulus type, with aphids generally recovering back from drops in response to the first standardized stimulus quicker than they recovered after dropping from a predator, as predicted (see Table 1). The slower recovery rate following predatorinduced drops may be due to aphids exhibiting a different behavioral pattern once activity was resumed. Wohlers (1981) found that pea aphids dislodged by foraging predators exhibited longer periods of running behavior before searching for and climbing plant models than those dislodged by touch alone. Potato aphids may similarly switch to searching behavior more rapidly following tactile stimulus-induced drops compared to predator-induced drops. In a study by Phelan et al. (1976) involving several aphid species (including potato aphids), aphids dislodged by mechanical stimuli oriented rapidly to vertical dowels while those dislodged by exposure to alarm pheromone increased their rate of locomotion, travelled greater distances and changed direction less often. As to the adaptive value of different recovery behaviors, this current study was limited by its inability to track specific paths of movement on the substrate and its consideration of only instances where aphids recovered back to their original host plants. Studies such as those by Phelan et al. (1976) and Wohlers (1981) tested aphids in arenas with many plant models and 
suggest, for example, that running behavior following predator-induced drops may serve to divert individuals away from original hosts that are dangerously infested with predators. Different triggers of dropping appear to have different consequences for postdropping locomotion, likely influencing the energetic, time and mortality risk costs of dropping (Losey and Denno 1998a, b; Nelson 2007; Agabiti et al. 2016; Humphreys and Ruxton 2019) as well as which plants aphids eventually recover to (Ben-Ari et al. 2015). However, more research is needed into the specific behaviors that potato aphids exhibit during their recovery time in order to fully understand any adaptive value they hold and their fitness consequences in different contexts. It is also interesting to note that while recovery rates back to host seedlings did seem to vary depending on the nature of the stimulus (suggesting some degree of discrimination between the stimuli) TI duration did not significantly differ following drops due to contact with a predator compared to contact from the standardized stimulus. The significance of the different time aphids spend exhibiting TI and the adaptive value of the behavior under different circumstances is not well understood (Wohlers 1981; Bilska et al. 2018), and so future research investigating the relative importance of different stimuli and other selective pressures on variations in both TI and post-TI locomotion will help to unpack this.

The recovery rate of potato aphids that returned to their original host seedling after dropping did not appear to be significantly affected by any of the other variables explored in this experiment. In contrast to our prediction (see Table 1), the different textures and grain structures of the sand and soil in this experiment did not generate significant differences in recovery rate once aphids had resumed activity; it was predicted that the looser sand substrate would present a greater challenge to aphid recovery than the more compacted composition of soil. Throughout assays the experimenter noted that aphids' ease of mobility did not consistently vary between the two substrate types - both the loosely-packed sand grains and the compacted chunks of soil occasionally caused some individuals temporary issues in locomotion, but mostly aphids appeared to travel with few problems. Substrate may be a more significant issue for post-dropping recovery if younger instars of aphid were tested, as their locomotion is more likely to be impeded by features like stones or grains of soil
(Roitberg et al. 1979). Closer study of the effects of the physical qualities of more varying substrates on aphid locomotion might be a useful avenue for future research. Although we did not base our prediction regarding the effect of substrate on recovery rate on its likely temperature or moisture content, it is worth reiterating that these features were not quantified or controlled for in this present study. While ambient temperature appeared to be a key influencer of how vulnerable aphids were to hot and dry conditions and/or how active individuals were, further work is needed to fully understand the contribution of different substrates under different conditions to both of these factors, and the effect of different conditions on the physical characteristics of the substrate itself as the surface over which aphids must travel. For example, following a spell of heavy rain a soil substrate may retain a lot more moisture compared to a sand substrate, potentially enabling dropped aphids to survive for a longer period of time before returning to a host plant; but interactions between substrate moisture content, substrate temperature, and ambient temperature and their influence on aphid behaviors and locomotion abilities need to be explored, including for a wider range of more natural substrate types. Neither the maximum height of the seedlings aphids dropped from, nor the horizontal distance aphids landed from their host seedling had a significant effect on their recovery rate back to that seedling. Theoretically, if insects of a particular species preferred to feed in locations higher up plants, we might predict that their recovery rates would be more rapid following drops from taller plants as it would take them longer to re-settle at an appropriate feeding location and they would be selected to minimize unnecessary time spent off-plant. However, in this study the range of maximum heights of seedlings was not controlled and did not vary greatly, and so we had anticipated that they might not affect recovery rate (Table 1). Further, we did not record the within-plant vertical feeding locations aphids dropped from. Concerning horizontal distance dropped, we had predicted that aphids that dropped a greater distance away might experience stronger selection to decrease the length of time they spent exposed to the risks associated with being off-plant [e.g. (Losey and Denno 1998a, b; Nelson 2007; Agabiti et al. 2016; Humphreys and Ruxton 2019)], as returning to the seedling would unavoidably take longer (Table 1). As with the TI duration 
findings, we think there are alternative variables than horizontal distance dropped that are probably more important to dropped individuals' assessment of risk on the substrate and post-dropping behavior.

\section{Mitigation of Dropping/Re-Clinging/Instant Recovery}

Most drops that preceded re-clinging were triggered by the standardized stimulus rather than predators; this was likely due to the relative infrequency of contact with predators (and subsequent dropping) compared with the standardized stimulus. Our consideration of seedling position was concerned with the type of plant section an aphid was located on rather than the vertical position up a plant, and - in agreement with our prediction (see Table 1) - all but one case of instant recovery followed drops from the leaf undersides. Leaf undersides are more likely to overhang other plant sections than the thinner and more vertical structures of plants, therefore they could be selected by aphids in order to increase their likelihood of instant recovery if dropping becomes necessary; the relative extent of underlying foliage cover was not recorded as part of this experiment, though. Further, or alternatively, this trend may instead be accounted for by a general tendency of aphids to settle on leaf undersides, perhaps as the most productive feeding sites and/or the sites most sheltered from predator detection and abiotic stress, as the vast majority of aphids settled on leaf undersides (rather than leaf upper sides, stems or petioles) throughout the experiment.

Considering plant factors, the likelihood of aphids re-clinging was not affected by seedling age, contrary to our prediction (see Table 1). But age was identified by the experimenter as not being a reliable measure of foliage cover or relative seedling height across assays. However, maximum height of seedlings (similar to the "field height" measure used by Meresman et al. 2017) did appear to influence the occurrence of instant recovery in potato aphids as predicted (see Table 1), with cases of post-drop re-clinging occurring significantly more commonly on pots with taller seedlings than shorter seedlings. This corresponds with previous findings that falling from greater heights increases aphids' abilities to successfully adopt an aerial righting posture (Ribak et al. 2013) and increases the likelihood of aphids re-clinging to underlying plant material (Meresman et al. 2017). Neither the timing of potato aphids' aerial righting nor seedlings' foliage cover as viewed in vertical direction from above were included in this current study. However, the likelihood of aphids falling from objectively higher positions is greater on taller seedlings and we would predict that foliage cover would be greater on taller seedlings, which have presumably grown more - outwards as well as upwards. Plant height and architecture could therefore be important to mitigating the costs of dropping for aphids. In pea aphids, for example, Meresman et al. (2017) have previously demonstrated that re-clinging success is positively dependent on the amount of underlying plant material available for re-clinging and plant height. Particular leaf shapes and plant architecture also appear to mitigate the costs of dropping for non-aphid insect species (Matsubara and Sugiura 2018). Further studies quantifying factors such as foliage cover, plant architecture, and specific pre-dropping positions within individual plants will be valuable to understanding the behavioral ecology of potato aphids and all other species for which post-drop instant recovery remains a relatively new and underappreciated topic. Future work should also consider separating out factors such as plant age and plant height that change concurrently over time, to see how they relate to foliage cover and the likelihood of instant recovery.

\section{Conclusion}

Through exploring the post-dropping behavior of potato aphids, an agriculturally-important species that has not received much attention regarding its antipredator defenses, this study identified several factors that could have an important influence on tonic immobility, recovery rate, and the likelihood of instant recovery through re-clinging. Future studies with finer-scale measurement and control of key variables such as temperature might reveal even stronger correlations with TI duration and recovery rate. The heights from which aphids dropped could also usefully be investigated more closely in future work. Studies purpose-designed to control the height dropped by aphids [e.g., Niku (1975)] or that have access to sophisticated technology to track distance fallen - rather than using maximum height of seedlings in a pot as this study did - might find significant correlations with TI duration or recovery rate, or a 
stronger relationship with the likelihood of re-clinging. Nonetheless, despite the relatively simple measures of temperature and seedling height used in this study, significant correlations in the predicted effect directions were found. This indicates that these relationships are highly important, and likely to recur in future studies with more precise measures. We certainly hope that this exploratory study will inspire researchers to design studies to address specific hypotheses about post-dropping behavior, and to do so for a wider range of species than has been investigated thus far.

Dropping is an antipredator behavior that carries nuanced short- and long-term consequences for prey and, as such, dropping is often used in a flexible way in order to minimize the cost (Humphreys and Ruxton 2019). The results of this current study, alongside previous work, indicate that post-dropping behaviors can also be complex and utilized in a nuanced way. For example, given that water loss, lost feeding time, and the risk of mortality from ground predators are obvious costs of slow recovery after dropping to the substrate (Losey and Denno 1998a, b; Nelson 2007; Agabiti et al. 2016; Humphreys and Ruxton 2019), it might be assumed that individuals would travel back to a plant as quickly as possible. However, the process of falling some distance could well attract nearby mobile visual predators, and therefore a short display of TI could be important to reduce localization of prey (Miyatake et al. 2009). Such TI itself, though, increases time off-plant and will carry a greater risk of desiccation when the environment is hotter and drier; though the humidity and moisture content of the environment and substrate were not explored in this current study, they should be considered in future, purpose-designed work. Immediate return to plants, therefore, might not always be the most adaptive option, but aphids must trade-off conflicting pressures associated with post-drop recovery. The easiest way for aphids to mitigate any costs of dropping (while still evading an approaching threat on the plant) is to re-cling to underlying plant material, but this is only possible under certain conditions. While the fitness of aphids in the wild will be determined primarily through repeated rather than one-off predator encounters, developing understanding of the behavioral variation following dropping defense under different conditions will allow the prediction of longer-term and larger-scale consequences for both prey and predator populations and their broader ecologies.

Acknowledgements We thank two anonymous referees for their helpful and insightful comments. RKH thanks the University of St Andrews and the Perry Foundation for funding. AJK is supported by the strategic research program funded by the Scottish Government's Rural and Environment Science and Analytical Services Division. We are very grateful to several members of the James Hutton Institute (Dundee), whose efforts made this work possible: Carolyn Mitchell for all her help with insect rearing (both in terms of practical work and guidance), Jim Wilde for the supply and maintenance of plant material, and Dave Gray for technical assistance with the Snijders Scientific MicroClima growth chamber used to maintain insects. Additional thanks go to Gaynor Malloch (James Hutton Institute, Dundee), Daniel Leybourne, Desiré Macheda, and Pilar Morera Margarit for advice on and assistance with aphid rearing, and to Elvyra Bulavaite for advice on maintenance of ladybirds and lacewings. Further thanks go to the James Hutton Institute (Dundee) for supplying the potato aphid lines used in experiments.

Authors' Contributions All authors contributed to the study conception and design. Material preparation, data collection and analysis were performed by Rosalind K. Humphreys. The first draft of the manuscript was written by Rosalind K. Humphreys and all authors commented on previous versions of the manuscript. All authors read and approved the final manuscript.

Funding RKH is supported by funding from the University of St Andrews and the Perry Foundation. AJK is supported by the strategic research program funded by the Scottish Government's Rural and Environment Science and Analytical Services Division.

Data Availability Data can be made available upon request.

Code Availability Not applicable.

\section{Declarations}

Conflicts of Interest/Competing Interests The authors have no conflicts of interest to declare that are relevant to the content of this article.

Open Access This article is licensed under a Creative Commons Attribution 4.0 International License, which permits use, sharing, adaptation, distribution and reproduction in any medium or format, as long as you give appropriate credit to the original author(s) and the source, provide a link to the Creative Commons licence, and indicate if changes were made. The images or other third party material in this article are included in the article's Creative Commons licence, unless indicated otherwise in a credit line to the material. If material is not included in the article's Creative Commons licence and your intended use is not permitted by statutory regulation or exceeds the permitted use, you will need to obtain permission directly 
from the copyright holder. To view a copy of this licence, visit http://creativecommons.org/licenses/by/4.0/.

\section{References}

Agabiti B, Wassenaar RJ, Winder L (2016) Dropping behaviour of pea aphid nymphs increases their development time and reduces their reproductive capacity as adults. PeerJ 4:e2236

AHDB (2015) Encyclopaedia of pests and natural enemies in field crops. Agriculture and Horticulture Development Board, Warwickshire

Ben-Ari M, Gish M, Inbar M (2015) Walking aphids can partake in within-field dispersal to distant plants. Basic Appl Ecol 16:162-171

Bilska A, Francikowski J, Wyglenda A, Masłowski A, Kaszyca N, Depa $Ł$ (2018) Aphids playing possum - defensive or mutualistic response? J Insect Behav 31:42-53

Broadbent L, Hollings M (1951) The influence of heat on some aphids. Ann Appl Biol 38:577-581

Dill LM, Fraser AHG, Roitberg BD (1990) The economics of escape behavior in the pea aphid, Acrythosiphon pisum. Oecologia 83:473-478

Friard O, Gamba M (2016) BORIS: a free, versatile opensource event-logging software for video/audio coding and live observations. Methods Ecol Evol 7:1325-1330

Humphreys RK, Ruxton GD (2018) A review of thanatosis (death feigning) as an anti-predator behaviour. Behav Ecol Sociobiol 72:22

Humphreys RK, Ruxton GD (2019) Dropping to escape: a review of an under-appreciated antipredator defence. Biol Rev 94:575-589

Humphreys RK, Ruxton GD, Karley AJ (2021) Drop when the stakes are high: adaptive, flexible use of dropping behaviour by aphids. Behaviour 158:603-623

Jusufi A, Zeng Y, Full RJ, Dudley R (2011) Aerial righting reflexes in flightless animals. Integr Comp Biol 51:937-943

Losey JE, Denno RF (1998a) The escape response of pea aphids to foliar-foraging predators: factors affecting dropping behaviour. Ecol Entomol 23:53-61

Losey JE, Denno RF (1998b) Interspecific variation in the escape responses of aphids: effect on risk of predation from foliar-foraging and ground-foraging predators. Oecologia 115:245-252

Matsubara S, Sugiura S (2018) Host plant architecture affects the costs of dropping behaviour in Phaedon brassicae (Coleoptera: Chrysomelidae). Appl Entomol Zoolog 53:501-508

Meresman Y, Ribak G, Weihs D, Inbar M (2014) The stimuli evoking the aerial-righting posture of falling pea aphids. $J$ Exp Biol 217:3504-3511

Meresman Y, Ben-Ari M, Inbar M (2017) Turning in mid-air allows aphids that flee the plant to avoid reaching the risky ground. Integr Zool 12:409-420
Miyatake T, Okada K, Harano T (2008) Negative relationship between ambient temperature and death-feigning intensity in adult Callosobruchus maculatus and Callosobruchus chinensis. Physiol Entomol 33:83-88

Miyatake T, Nakayama S, Nishi Y, Nakajima S (2009) Tonically immobilized selfish prey can survive by sacrificing others. P Roy Soc B-Biol Sci 276:2763-2767

Nelson EH (2007) Predator avoidance behavior in the pea aphid: costs, frequency, and population consequences. Oecologia 151:22-32

Niku B (1975) Verhalten und fruchtbarkeit ungeflügelter erbsenläuse (Acyrthosiphon pisum) nach einer fallreaktion. Entomol Exp Appl 18:17-30

Niku B (1976) Some consequences of the drop reaction of Acyrthosiphon pisum for the larvae of Syrphus corollae. Entomophaga 21:257-264

Phelan PL, Montgomery ME, Nault LR (1976) Orientation and locomotion of apterous aphids dislodged from their hosts by alarm pheromone. Ann Entomol Soc Am 69:1153-1156

R Core Team (2020) R: A language and environment for statistical computing. R Foundation for Statistical Computing,Vienna

Ribak G, Gish M, Weihs D, Inbar M (2013) Adaptive aerial righting during the escape dropping of wingless pea aphids. Curr Biol 23:R102-R103

Rogers SM, Simpson SJ (2014) Thanatosis. Curr Biol 24:R1031-R1033

Roitberg BD, Myers JH (1979) Behavioural and physiological adaptations of pea aphids (Homoptera: Aphididae) to high ground temperatures and predator disturbance. Can Entomol 111:515-519

Roitberg BD, Myers JH, Frazer BD (1979) The influence of predators on the movement of apterous pea aphids between plants. J Anim Ecol 48:111-122

Ruth WE, McNew RW, Caves DW, Elkenbary RD (1975) Greenbugs (Hom.: Aphididae) forced from host plants by Lysiphlehus testaceipes (Hym.: Braconidae). Entomophaga 20:65-71

van Emden HF, Harrington R (eds) (2007) Aphids as crop pests. CABI, Trowbridge

Wohlers P (1981) Effects of the alarm pheromone (E)- $\beta$ farnesense on dispersal behaviour of the pea aphid Acyrthosiphon pisum. Entomol Exp Appl 29:117-124

Yanoviak SP, Kaspari M, Dudley R (2009) Gliding hexapods and the origins of insect aerial behaviour. Biol Lett 5:510-512

Zeng Y, Lam K, Chen YX, Gong MS, Xu ZY, Dudley R (2017) Biomechanics of aerial righting in wingless nymphal stick insects. Interface Focus 7:12

Publisher's Note Springer Nature remains neutral with regard to jurisdictional claims in published maps and institutional affiliations. 